\title{
Synthesis of New Chiral Crown Ethers Incorporating Two Different Chiral Units and "Matched/Mismatched" Effect of the Two Chiral Units on the Chiral Recognition
}

\author{
Yoon Jae Cho, Hee Jung Choi, and Myung Ho Hyun * \\ Department of Chemistry and Chemistry Institute for Funtional Materials, Pusan National University, Busan 609-735, Korea \\ "E-mail:mhhyun@pisanackr \\ Received July 30,2007
}

Key Words : Chiral crown ether, Chiral stationary phase, Matched/mismatched effect

Chiral crown ethers have been widely applied as chiral selectors in differentiating the two enantiomers of racemic compounds containing a primary amino group. ${ }^{1}$ Especially chiral crown ethers have been quite useful as chiral selectors of liquid chromatographic chiral stationary phases (CSPs) for the recognition of the two enantiomers of racemic primary amino compounds. Among a large number of chiral crown ethers, two types have been most successfully utilized as liquid chromatographic CSPs. The first type is chiral crown ethers incorporating chiral 1, I'-binaphthyl unit, which were first developed by Cram. ${ }^{2}$ Especially, chiral crown ethers such as bis-( $1,1^{\prime}$-binaphthy])-22-crown-6 immobilized on silica gel or polystyrene were used as CSPs in separating the enantiomers of racemic $\alpha$-amino acids and their derivatives in the late $1970 \mathrm{~s}$ by Cram and coworkers. ${ }^{3}$ Afterward, chiral crown ethers related to (3,3-diphenyl-1,1'binaphthyl)-20-crown-6 (1, Fig. I) dynamically coated on octadecyl silica gel ${ }^{+}$or covalently bonded to silica gel ${ }^{5}$ were developed as liquid chromatographic CSPs and successfully utilized in the resolution of racemic $\alpha$-amino acids and other racemic compounds containing a primary amino group.

The second type of chiral crown ether successfully utilized as a chiral selector of CSPs is the one incorporating two tartaric acid units, (+)-(18-crown-6)-2,3,11,12-tetracarboxylic acid (2, Fig. 1), which was first synthesized by Lehn and coworkers. ${ }^{6}$ Initially, (+)-(18-crown-6)-2,3,11,12-tetracarboxylic acid (2) was utilized as a chiral selector for the chiral separation by capillary electrophoresis. ${ }^{7}$ The use of (+)-(18-crown-6)-2,3,11,12-tetracarboxylic acid (2) was then extended as a chiral selector of liquid chromatographic CSPs. Liquid chromatographic CSPs based on $(+)-(18-$ crown-6)-2,3,11,12-tetracarboxylic acid (2) were very successful in the resolution of various racemic primary

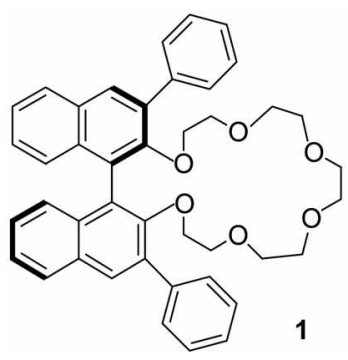<smiles>O=C(O)C1OCCOCCO[C@H](C(=O)O)[C@@H](C(=O)O)OCCOCCO1</smiles>

2 amino compounds ${ }^{8}$ or racemic non-primary amino compounds. ${ }^{9}$ In addition, (+)-(18-crown-6)-2,3,11,12-tetracarboxylic acid (2) was utilized as an NMR chiral solvating agent. $^{10}$

However, chiral crown ethers incorporating both chiral 3,3-diphenyl-1,I-binaphthyl and tartaric acid unit have never been known to the best of our knowledge. Chiral crown ethers incorporating both chiral 3,3'-dipheny]-1,1'binaphthyl and tartaric acid unit are expected to show characteristics somewhat different from chiral crown ether 1 or $\mathbf{2}$ in the chiral recognition. In this study, we wish to report the synthesis of novel chiral crown ethers incorporating both chiral 3,3-diphenyl-1, ['-binaphthyl and tartaric acid unit and their applications as chiral selectors of liquid chromatographic CSPs.

We prepared actually two diastereomeric chiral crown ethers, $(R, R, R)-3$ and $(R, S, S)-3$ (Fig. 2), incorporating both $(R)$-3,3'-diphenyl-1,1'-birlaphthyl and $(R, R)$ - or $(S, S)$-tartaric acid unit as chiral barriers. Combination of the two different chiral units as chiral barriers is expected to endow the two diastereomeric chiral crown ethers with characteristic chiral recognition ability according to the stereochemistry of the two chiral units.

Synthesis of the two diastereomeric chiral crown ethers was successfully performed as shown in Scheme 1. Diols $(R, R)-4$ and $(S, S)-4$ prepared from commercially available $(R, R)$ - and $(S, S)-N, N, N^{\prime}, N^{\prime}$-tetramethyltartaramide respectively via the known procedure ${ }^{11}$ were converted into their tosyl derivatives, $(R, R)-5$ and $(S, S)-5$. Tosylation of $(R, R)-\mathbf{4}$ or $(S, S)-4$ was most successful when a small amount of $4-$ (dimethylamino)pyridine (DMAP) was added to the reaction mixture. The cyclization of tosyl derivative $(R, R)-5$ or $(S, S)$ -

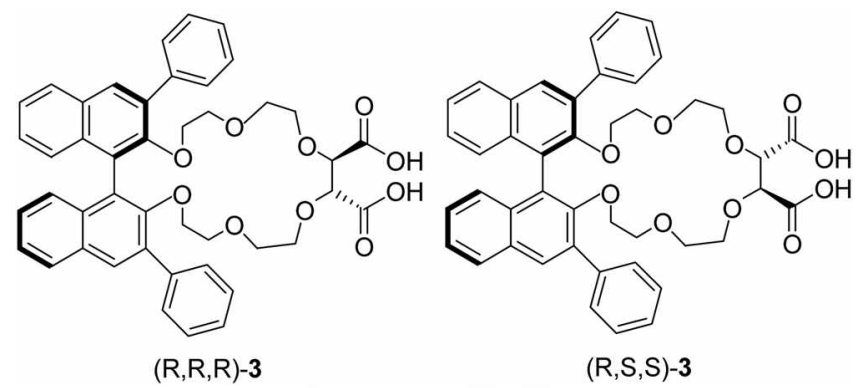

Figure 2. Structures of diastereomenc chral crown ethers $(R, R, R)$ 3 and $(R, S, S)-3$.

Figure 1. Structures of chiral crown ethers 1 and 2. 
<smiles>CC(=O)C(C(=O)OCCOCCO)[C@@H](OCCO)C(C)=O</smiles>

$(\mathrm{R}, \mathrm{R})-4$

$(\mathrm{S}, \mathrm{S})-4$

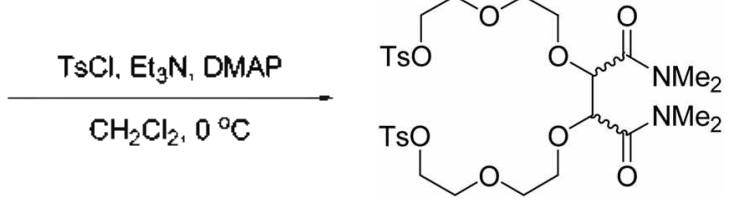

(R,R)-5 (92\%)

(S,S)-5 (90\%)
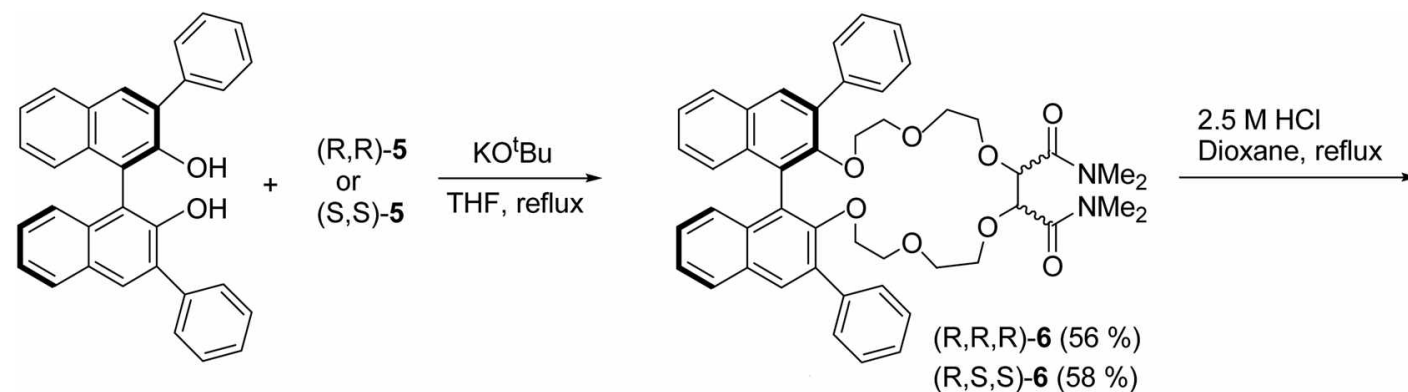

$(R, R, R)-3(94 \%)$
and
$(R, S, S)-3(95 \%)$

$(\mathrm{R}, \mathrm{S}, \mathrm{S})-6(58 \%)$

Scheme 1. Synthesis of chiral crown ethers $(R, R, R)-3$ and $(R, S, S)-3$.
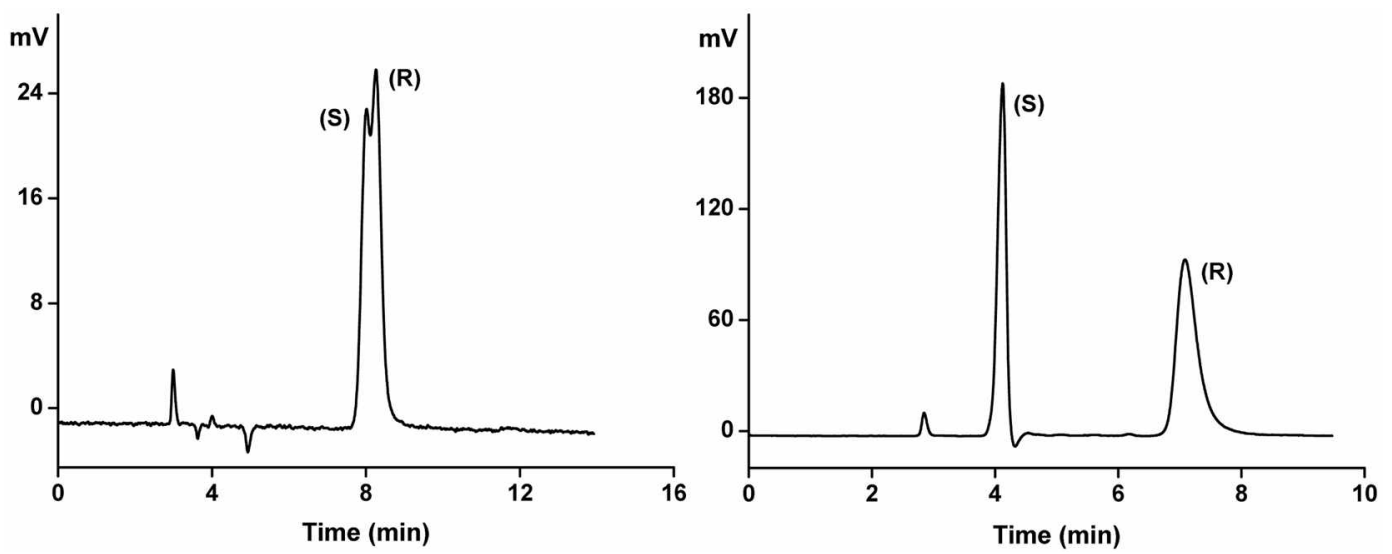

Figure 3. Representative chromatograuns for the resolution of phenylglycine $n$-propylamide on $(R, R, R)-3-\mathrm{CSP}$ (Icf) and on $(R, S, S)$-3-CSP (right). Mobile phase: ethanol/hexane/trifhloroacetic acid/water, 30:70:0.5:0.2. Flow rate: $0.5 \mathrm{~mL} / \mathrm{min}$. Detection: $210 \mathrm{~nm}$ UV. Temperature: $20^{\circ} \mathrm{C}$.

5 with $(R)-3,3^{\prime}$-diphenyl-1,1'-bi-2-naphthol prepared from $(R)-1,1^{\prime}$-bi-2-naphthol via the known procedure ${ }^{12}$ to form chiral crown ethers $(R, R, R)-6$ and $(R, S, S)-6$ was quite successful with the use of potassium tert-butoxide as a base in THF at reflux temperature. Finally, each of chiral crown ethers $(R, R, R)-6$ and $(R, S, S)-6$ was treated with $2.5 \mathrm{M} \mathrm{HCl}$ solution in dioxane at reflux temperature to afford chiral crown ethers $(R, R, R)-\mathbf{3}$ and $(R, S, S)-\mathbf{3}$.

The NMR study to evaluate the two diastereomeric chiral crown ethers for their chiral recognition ability was not successful yet. Altematively, we simply attached each of the two chiral crown ethers through one carboxylic acid group to aminopropylsilica gel and the resulting CSPs were briefly tested for their chiral recognition ability. ${ }^{13}$ The representative chromatograms are presented in Figure 3. As shown in Figure 3 , the CSP derived from $(R, S, S)-3$ [ $[(R, S, S)-3$-CSP $]$ shows excellent chiral recognition (separation factor $\alpha=$ $3.77)$, but the CSP derived from $(R, R, R)-\mathbf{3}[(R, R, R)-3$-CSP $]$ shows very poor chiral recognition $(\alpha=1.05)$. The resolution result on $(R, S, S)-3$-CSP is even greater than that on a CSP based on chiral crown ether $1(\alpha=1.80$ under an identical condition) or on a CSP based on chiral crown ether $2\left(\alpha=2.67\right.$ under a reverse phase condition) ${ }^{1+}$ From these results, we conclude that combination of $(R)$-3,3'-diphenyl1,1'-binaphthyl and $(S, S)$-tartaric acid unit shows a "matched" effect on the chiral recognition while combination of $(R)$-3,3-diphenyl-1,1'-binaphthyl and $(R, R)$-tartaric acid unit shows a "mismatched" effect. ${ }^{15}$

In summary we have synthesized two new diastereomeric chiral crown ethers incorporating both chiral 3,3'-diphenyl1,1'-binaphtyl and tartaric acid unit as chiral barriers. The two chiral crown ethers bonded to aminopropylsilica gel were demonstrated to show different chiral recognition ability according to the stereochemistry of the two chiral units incorporated. From these results, the two chiral units were expected to show "matched/mismatched" effect on the chiral recognition of the two diastereomeric chiral crown ethers.

\section{Experimental Section}

Preparation of $(\boldsymbol{R}, \boldsymbol{R})$-5. $p$-Toluenesulfonyl chloride (1.70 $\mathrm{g}, 8.9 \mathrm{mmol})$ was slowly added to a solution of $(R, R)-4(1.42$ 
$\mathrm{g}, 3.72 \mathrm{mmol})$, triethylamine $(1.24 \mathrm{~mL}, 8.9 \mathrm{mmol})$ and $4-$ (dimethylamino)pyridine (DMAP) $(24 \mathrm{mg}, 0.2 \mathrm{mmol}$ ) in methylene chloride $\left(15 \mathrm{~mL}\right.$ ) at $0^{\circ} \mathrm{C}$. After stirring for $24 \mathrm{~h}$ at $\mathrm{rt}$, the reaction mixture was washed with $0.5 \mathrm{M} \mathrm{NaOH}$ solution, $0.5 \mathrm{M} \mathrm{HCl}$ solution and then brine. The organic solution was dried over anhydrous $\mathrm{Na}_{2} \mathrm{SO}_{4}$ and concentrated. Chromatography of the residue over silica gel (THF/ Hexane, $1: 1, \mathrm{v} / \mathrm{v})$ afforded $(R, R)-5(2.30 \mathrm{~g}, 92 \%)$ as a colorless oily material. $[\alpha]_{\mathrm{D}}^{26}+47.2\left(\mathrm{c} 0.5, \mathrm{CHCl}_{3}\right)$. IR $(\mathrm{KBr})$ $3064,2931,2875,1647,1507,1457 \mathrm{~cm}^{-1}$. ${ }^{1} \mathrm{H}$ NMR $(500$ $\left.\mathrm{MHz}, \mathrm{CDCl}_{3}\right): \delta 2.46(\mathrm{~s}, 6 \mathrm{H}), 2.93(\mathrm{~s}, 6 \mathrm{H}), 3.16(\mathrm{~s}, 6 \mathrm{H}) 3.55-$ $3.63(\mathrm{~m}, 4 \mathrm{H}), 3.66-3.68(\mathrm{~m}, 4 \mathrm{H}), 3.69-3.78(\mathrm{~m}, 4 \mathrm{H}), 4.14-$ $4.16(\mathrm{~m}, 4 \mathrm{H}), 4.78(\mathrm{~s}, 2 \mathrm{H}), 7.36(\mathrm{~d}, 4 \mathrm{H}, J=7.9 \mathrm{~Hz}), 7.80(\mathrm{~d}$, $4 \mathrm{H}, J=8.3 \mathrm{~Hz}) .{ }^{13} \mathrm{C} \mathrm{NMR}\left(125 \mathrm{MHz}, \mathrm{CDCl}_{3}\right) \delta 21.8,36.0$, $37.6,68.9,69.2,69.5,71.0,77.5,128.2,130.1,133.2,145.1$, 169.6. HRMS (FAB+): $[\mathrm{M}+\mathrm{H}]^{+}$calcd for $\mathrm{C}_{30} \mathrm{H}_{45} \mathrm{~N}_{2} \mathrm{O}_{12} \mathrm{~S}_{2}$, 689.2414; found, 689.2411. (S,S)-5 was prepared via the identical procedure $(90 \%) .[a]_{\mathrm{D}}^{26}-46.8\left(\mathrm{c} 0.5, \mathrm{CHCl}_{3}\right)$.

Preparation of $(\boldsymbol{R}, \boldsymbol{R}, \boldsymbol{R})-\mathbf{6}$. A solution of $(R)-3,3^{\prime}$-diphenyl-1,1'-bi-2-naphthol $(0.65 \mathrm{~g}, 1.48 \mathrm{mmol})$, which was prepared from $(R)-1,1^{\prime}$-bi-2-naphthol via the known procedure, ${ }^{13}$ in THF $(50 \mathrm{~mL})$ was slowly added to a solution of potassium-tert-butoxide $(0.35 \mathrm{~g}, 3.11 \mathrm{mmol})$ in THF (50) $\mathrm{mL}$ ). The reaction mixture was stirred for $30 \mathrm{~min}$ at $\mathrm{rt}$ and then a solution of $(R, R)-5(1.02 \mathrm{~g}, 1.48 \mathrm{mmol})$ in THF $(50$ $\mathrm{mL}$ ) was slowly added. After stirring for $72 \mathrm{~h}$ at reflux temperature, the reaction mixture was rotary-evaporated and then the residue was dissolved in methylene chloride $(80$ $\mathrm{mL}$ ). The solution was washed with $0.5 \mathrm{M} \mathrm{NaOH}$ solution, $0.5 \mathrm{M} \mathrm{HCl}$ solution and then brine. The organic layer was dried over anhydrous $\mathrm{Na}_{2} \mathrm{SO}_{4}$ and concentrated. Chromatography of the residue over silica gel (methanol:ethyl acetate $=1: 5)$ afforded $(R, R, R)-6(0.68 \mathrm{~g}, 56 \%)$ as a colorless oily material. $[\alpha]_{\mathrm{D}}^{36}+93.7\left(\mathrm{c} 0.3, \mathrm{CHCl}_{3}\right)$. IR (KBr) 3055,2928 , $2870,1649,1496,1457,1418 \mathrm{~cm}^{-1}$. ${ }^{1} \mathrm{H}$ NMR $(500 \mathrm{MHz}$, $\left.\mathrm{CDCl}_{3}\right) \delta 2.91(\mathrm{~s}, 6 \mathrm{H}), 2.98-3.02\left(\mathrm{~m}, 2 \mathrm{H}, \mathrm{CH}_{2}\right), 3.11(\mathrm{~s}, 6 \mathrm{H})$, $3.12-3.21(\mathrm{~m}, 4 \mathrm{H}), 3.36-3.40(\mathrm{~m}, 2 \mathrm{H}), 3.48-3.54(\mathrm{~m}, 4 \mathrm{H})$, $3.57-3.61(\mathrm{~m}, 2 \mathrm{H}), 3.68-3.72(\mathrm{~m}, 2 \mathrm{H}), 4.76(\mathrm{~s}, 2 \mathrm{H}), 7.20(\mathrm{~d}$, $2 \mathrm{H}, J=8.0 \mathrm{~Hz}), 7.26-7.29(\mathrm{~m}, 2 \mathrm{H}), 7.39-7.44(\mathrm{~m}, 4 \mathrm{H}), 7.52$ $(\mathrm{t}, 4 \mathrm{H}, J=7.7 \mathrm{~Hz}), 7.79(\mathrm{~d}, 4 \mathrm{H}, J=7.0 \mathrm{~Hz}), 7.92(\mathrm{~d}, 2 \mathrm{H}, J=$ $8.0 \mathrm{~Hz}), 7.98(\mathrm{~s}, 2 \mathrm{H}) .{ }^{13} \mathrm{C} \mathrm{NMR}\left(125 \mathrm{MHz}, \mathrm{CDCl}_{3}\right) \delta 35.9$, $37.6,68.8,70.1,70.5,72.3,76.6,125.3,126.0,126.6,127.6$, $128.3,128.6,128.7,129.8,130.8,130.9,133.8,135.4$, 139.2, 153.7, 169.8. HRMS (FAB+): $[\mathrm{M}+\mathrm{H}]^{+}$calcd for $\mathrm{C}_{48} \mathrm{H}_{51} \mathrm{~N}_{2} \mathrm{O}_{8}, 783.3645$; found, 783.3649. $(R, S, S)-6$ was prepared via the identical procedure $(58 \%)$. $[\alpha]_{D}^{26}-38.5(\mathrm{c} 0.3$, $\left.\mathrm{CHCl}_{3}\right)$. IR (KBr) $3055,2930,2871,1650,1496,1418 \mathrm{~cm}^{-1}$. 'H NMR $\left(500 \mathrm{MHz}_{3} \mathrm{CDCl}_{3}\right) \delta 2.92(\mathrm{~s}, 6 \mathrm{H}), 3.04-3.08(\mathrm{~m}$, $2 \mathrm{H}), 3.12-3.16(\mathrm{~m}, 2 \mathrm{H}), 3.14(\mathrm{~s}, 6 \mathrm{H}), 3.25-3.27(\mathrm{~m}, 2 \mathrm{H})$, $3.35-3.38(\mathrm{~m}, 2 \mathrm{H}), 3.53-3.57(\mathrm{~m}, 4 \mathrm{H}), 3.61-3.62(\mathrm{~m}, 2 \mathrm{H})$, $3.69-3.73(\mathrm{~m}, 2 \mathrm{H}), 4.75(\mathrm{~s}, 2 \mathrm{H}), 7.19$ (d, $2 \mathrm{H}, J=8.5 \mathrm{~Hz}$ ), $7.26(\mathrm{~d}, 2 \mathrm{H}, J=6.9 \mathrm{~Hz}), 7.39-7.43(\mathrm{~m}, 4 \mathrm{H}), 7.50(\mathrm{t}, 4 \mathrm{H}, J=$ $7.6 \mathrm{~Hz}), 7.80(\mathrm{~d}, 4 \mathrm{H}, J=7.5 \mathrm{~Hz}), 7.92(\mathrm{~d}, 2 \mathrm{H}, J=8.0 \mathrm{~Hz})$, $7.99(\mathrm{~s}, 2 \mathrm{H}) .{ }^{13} \mathrm{C} \mathrm{NMR}\left(125 \mathrm{MHz}, \mathrm{CDCl}_{3}\right) \delta 35.9,37.5,69.3$, $70.1,70.3,72.1,76.9,125.3,126.0,126.6,127.7,128.3$, $128.6,128.7,129.8,130.7,131.0,133.8,135.4,139.1,153.6$, 169.7. HRMS (FAB+): $\left[\mathrm{M}+\mathrm{H}^{+}\right.$calcd for $\mathrm{C}_{48} \mathrm{H}_{51} \mathrm{~N}_{2} \mathrm{O}_{8}$,
783.3645; found, 783.3647 .

Preparation of $(R, R, R)-3$. To a solution of crown ether $(R, R, R)-6(0.68 \mathrm{~g}, 0.87 \mathrm{mmol})$ in dioxane $(10 \mathrm{~mL})$ was added $2.5 \mathrm{M} \mathrm{HCl}$ solution $(2 \mathrm{~mL}$ ). After stirring for $24 \mathrm{~h}$ at reflux temperature, the reaction mixture was rotary-evaporated and then the residue was dissolved in $\mathrm{CHCl}_{3}(15 \mathrm{~mL})$. The organic solution was washed with $1.0 \mathrm{M} \mathrm{HCl}$ solution and then brine. The organic solution was dried over anhydrous $\mathrm{Na}_{2} \mathrm{SO}_{4}$ and then concentrated. Chromatography of the residue on silica gel (methanol: $\mathrm{CHCl}_{3}=1: 10, \mathrm{v} / \mathrm{v}$ ) and then crystallization from $\mathrm{CHCl}_{3}$ and hexane afforded $(R, R, R)-3$ $(0.60 \mathrm{~g}, 94 \%)$ as a white crystalline material: mp $247-250^{\circ} \mathrm{C}$ $\left(\mathrm{CHCl}_{3} /\right.$ hexane); $[a]_{\mathrm{D}}^{25}-113.8$ (c $\left.0.1, \mathrm{CHCl}_{3}\right)$. IR (KBr) $3421,3056,2932,2877,1604,1419 \mathrm{~cm}^{-1}$. 'H NMR $(500$ $\left.\mathrm{MHz}, \mathrm{CH}_{3} \mathrm{OH}-\mathrm{d}_{4}\right) \delta 2.68-2.70(\mathrm{~m}, 2 \mathrm{H}), 2.98-3.01(\mathrm{~m}, 2 \mathrm{H})$, 3.17-3.19 (m, 2H), 3.27-3.31 (m, 2H), 3.36-3.39 (m, 2H), $3.55-3.58(\mathrm{~m}, 4 \mathrm{H}), 3.83-3.86(\mathrm{~m}, 2 \mathrm{H}), 4.33(\mathrm{~s}, 2 \mathrm{H}), 7.00(\mathrm{~d}$, $2 \mathrm{H}, J=8.5 \mathrm{~Hz}), 7.27(\mathrm{t}, 2 \mathrm{H}, J=7.7 \mathrm{~Hz}), 7.45(\mathrm{td}, 4 \mathrm{H}, J=7.3$ $\mathrm{Hz}, J=11.0 \mathrm{~Hz}), 7.65(\mathrm{t}, 4 \mathrm{H}, J=7.7 \mathrm{~Hz}), 7.77(\mathrm{~d}, 4 \mathrm{H}, J=$ $7.7 \mathrm{~Hz}), 8.02(\mathrm{~d}, 2 \mathrm{H}, J=8.2 \mathrm{~Hz}), 8.10(\mathrm{~s}, 2 \mathrm{H}) .{ }^{13} \mathrm{C}$ NMR (125 MHz, $\left.\mathrm{CH}_{3} \mathrm{OH}-\mathrm{d}_{4}\right) \delta 68.9,69.1,69.3,71.0,80.8,125.5$, $125.7,125.8,126.9,127.6,128.5,129.1,129.9,130.8$, $131.7,133.6,136.2,138.0,152.8,174.4$. HRMS (FAB+): $[\mathrm{M}+\mathrm{Na}]^{+}$calcd for $\mathrm{C}_{44} \mathrm{H}_{40} \mathrm{O}_{10} \mathrm{Na}, 751.2519$; found, 751.2518. $(R, S, S)$-3 was prepared via the identical procedure $(95 \%)$ : mp $209-212^{\circ} \mathrm{C}$. $[\alpha]_{\mathrm{D}}^{27}-29.2\left(\mathrm{c} 0.1, \mathrm{CHCl}_{3}\right)$. (KBr) 3419 , $3055,2930,2875,1616,1420 \mathrm{~cm}^{-1}$. 'H NMR $(500 \mathrm{MHz}$, $\left.\mathrm{CH}_{3} \mathrm{OH}-\mathrm{d}_{4}\right) \delta 2.82-2.85(\mathrm{~m}, 2 \mathrm{H}), 3.25-3.34(\mathrm{~m}, 6 \mathrm{H}), 3.40-$ $3.43(\mathrm{~m}, 2 \mathrm{H}), 3.49-3.53(\mathrm{~m}, 2 \mathrm{H}), 3.60-3.63(\mathrm{~m}, 2 \mathrm{H}), 3.76-$ $3.80(\mathrm{~m}, 2 \mathrm{H}), 4.28(\mathrm{~s}, 1 \mathrm{H}), 7.04(\mathrm{~d}, 2 \mathrm{H}, J=8.5 \mathrm{~Hz}), 7.25-$ $7.28(\mathrm{~m}, 2 \mathrm{H}), 7.41-7.46(\mathrm{~m}, 4 \mathrm{H}), 7.61(\mathrm{t}, 4 \mathrm{H}, J=7.8 \mathrm{~Hz})$, $7.76-7.78(\mathrm{~m}, 4 \mathrm{H}), 8.00(\mathrm{~d}, 2 \mathrm{H}, J=8.2 \mathrm{~Hz}), 8.06(\mathrm{~s}, 2 \mathrm{H})$. ${ }^{13} \mathrm{C}$ NMR $\left(125 \mathrm{MHz}, \mathrm{CH}_{3} \mathrm{OH}-\mathrm{d}_{4}\right) \delta 68.5,69.1,69.2,71.0$, $80.3,125.5,125.6,125.7,126.7,127.6,128.4,128.9,129.7$, $130.8,131.6,133.7,135.9,138.4,152.7,173.1$. HRMS $(\mathrm{FAB}+):[\mathrm{M}+\mathrm{Na}]^{+}$calcd for $\mathrm{C}_{44} \mathrm{H}_{40} \mathrm{O}_{10} \mathrm{Na}, 751.2519$; found, 751.2522 .

Acknowledgements. This work has been supported by grants-in-aid for the National Core Research Center Program from MOST/KOSEF (No. R15-2006-022-03001-0).

\section{References and Notes}

I. For selected reviews see: (a) Cram, D. J. Science 1988, 240, 760. (b) Zhang, X. X.; Bradshaw, J. S.; Izatl, R. M. Chem. Rev: 1997, 97,3313. (c) Hyun, M. H. Bull. Kor. Chem. Soc. 2005, 26, 1153.

2. Kyba, E. P.; Siegel, M. Gi; Sousa, L. R.; Sogah, G. D. Y.; Cram, D. J. J. Am. Chem. Soc. 1973, 95, 2691.

3. (a) Sousa, L. R.; Sogah, G. D. Y.; Hoffinan, D. H.; Cram, D. J. $J$. An. Chent. Soc, 1978, 100,4569 (b) Sogah, G. D. Y.; Cram, D. J. J. Am. Chem, Soc. 1979, 101, 3035 .

4. (a) Shinbo, T.; Yamaguchi, T.; Nishimura, K.; Sugiura, M. $J$. Chromatogr: 1987, 405, 145. (b) Shinbo, T.; Yamaguchi, T.; Yanagishita, H.; Kitamoto, D.; Sakaki, K.; Sugiura, M. $J$. Chromatogr 1992, 625, 101.

5. (a) Hyun, M. H.; Han, S. C.; Lipshutz, B. H.; Shin, Y.-J.; Welch, C. J. J. Chromatogr, $A$ 2001, 910, 359. (b) Hyun, M. H.; Han, \$. C.; Lipshutz, B. H.; Shin, Y.-J.; Welch, C. J. J. Chromatogr: $A$ 2002, 959, 75. (c) Hyun, M. H.; Min, H. J.; Cho, Y. J. $J$. 
Chrontatogr A 2003, 996, 233. (d) Hyun, M. H.; Tan, G; Cho, Y. J. Bionted. Chronlatogr. 2005, 19, 208. (e) Hyun, M. H.; Han, S. C.; Choi, H. J.; Kang. B. S.; Ha. H. J. J. Chronatogr. A 2007 , $1138,169$.

6. Behr, J.-P.; Girodeau, J.-M.; Hayward, R. C.; Lehn, J.-M.; Sauvage, J.-P. Hel. Chim. Acta 1980, 63, 2096.

7. For examples see: (a) Mori, Y.; Ueno, K.; Umeda, T. J. Chrontatogr $A$ 1997, 757, 328. (b) Cho, S. I.; Jung, H.; Chung, D. S. Electrophoresis $2000,21,3618$.

8. (a) Machida, Y,; Nishi, H.; Nakamura, K.; Nakai, H.; Sato, T. J. Chromatogra, $A$ 1998, 805,85 . (b) Hyun, M. H.; Jin, J. S.; Lee, W. J. Chromatogr: A 1998, 822, 155. (c) Hyun, M. H.; Jin, J. S.; Koo, H. J.; Lee, W. J. Chronatogr. A 1999, 837, 75. (d) Hyun, M. H.; Cho, Y. J.; Kim, J. A.; Jin, J. S. J. Chronatogr. A 2003, 984 , 163; (e) Hyun. M. H.; Kim, D. H. Chirulity 2004, 16, 294. (f) Hyun, M. H.; Cho, Y. J. J. Sep. Sci. 2005, 28, 31 . (g) Hyun, M. H.; Song, Y; Cho, Y. J.; Kim, D. H. J. Chromatogr: A 2006, 1108, 208. (h) Hyun, M. H.; Choi, H. J.; Kang, B. S.; Tan, G.; Cho, Y. J. Butl. Kor. Chem. Soc. 2006, 27, 1775. (i) Hyun, M. H.; Cho, Y. J.; Song, Y.; Choi, H. J.; Kang, B. S. Chirdity $2007,19,74$.

9. (a) Steffeck, R. J.; Zelechonok, Y.; Gahm, K. H. J. Chromatogr. $A$ 2002, 947, 301. (b) Zhang, D.; Li, F.; Kim. D. H.; Choi, H. J.; Hyun, M. H. J. Chromatogr: A 2005, 1083,89 . (c) Hyun, M. H.; Tan, G; Xue, J. Y. J. Chromatogr, A 2005, 1097, I88. (d) Tan, G.; Xue, J. Y.; Hyun, M. H. J. Sep. Sci. 2006, 29, 1407.

10. (a) Bang. E.; Jung, J.-W.; Lee, W.; Lee, D. W.; Lee, W. J. Chen.
Soc. Perkin Trans. 2 2001, 1685, (b) Machida, Y.; Kagawa, M.; Nishi, H. J. Pharnt. Bioned. Anal. 2003, 30, 1929. (c) Lovely, A. E.; Wenzel, T. J. J. Org. Chem. 2006, 71,9178.

I1. Fyles, T. M.; McGavin, C. A.; Whitfield, D. M. J. Org. Chen. $1984,49,753$

12. (a) Simonsen, K. B.; Gothelf, K. V; Jorgensen, K. A. J. Org. Chem, 1998, 63, 7536. (b) Wipd, P.; Jung, J.-K. J. Org. Chem. $2000,65,6319$.

13. The detailed study for the preparation of CSPs based on $(R, R, R)-3$ and $(R, S, S)-3$, their applications to the resolution of racemic primary amino compounds and the model study for the rationalization of the different chiral recognition ability of $(R, R, R)-\mathbf{3}$ and $\langle R, S, S\}-3$ are underway in our laboratory and will be reported elsewhere in the future.

14. Under an identical condition, the analyte was not eluted from a CSP based on chiral crown ether 2. Consequently, the reportedseparation factor under a reverse mobile phase condition is presented. Hyun, M. H.; Jin, J. S.; Lee, W. J. Chromatogr: $A$ 1998, 822,55 .

15. "Matched/mismatched" terminology has been used to describe the stereocontrol of chiral catalysts containing multiple stereocenters or in the formation of supramolecular complexes. For examples: (a) Heinriches, G; Kubik, S.; Lacour, J.; Vial, L. J. Org. Chem. 2005, 70, 4498. (b) Doyle, M. P.; Morgan, J. P.; Fettinger, J. C.; Zavalij, P. Y.; Colyer, J. T.; Timmons, D. J.; Carducci, M. D. J. Org. Chen. 2005, 70, 529l. 